\title{
La tuberculose au Canada - Résumé 2015
}

\author{
V Gallant ${ }^{1 *}$, V Duvvuri' ${ }^{1}$, M McGuire ${ }^{1}$
}

\section{Résumé}

Contexte : La tuberculose est un problème de santé mondial qui touche environ 10 millions de personnes chaque année. Au Canada, I'Agence de la santé publique du Canada (ASPC) surveille la tuberculose progressive au moyen du Système canadien de déclaration des cas de tuberculose (SCDCT).

Objectif : Faire le compte rendu et l'analyse du nombre de nouveaux cas de tuberculose et de cas de retraitement de la tuberculose déclarés au Canada en 2015. Les résultats sont discutés en fonction du contexte des données des années précédentes. Les résultats du traitement des cas diagnostiqués en 2014 sont également présentés.

Méthodologie : Le SCDCT est un système de surveillance fondé sur les cas qui conserve des données non nominatives sur les cas de tuberculose progressive. Les données sont recueillies et analysées par I'ASPC et validées par chaque province et territoire; aucun test statistique n'a été utilisé.

Résultats : Au total, 1639 cas de tuberculose progressive ont été déclarés en 2015, ce qui représente une légère augmentation du nombre de cas déclarés en 2014 (1 614) et une augmentation correspondante du taux d'incidence de 4,5 cas pour 100000 habitants à 4,6 cas pour 100000 habitants. Bien que le taux d'incidence de la tuberculose soit demeuré plus élevé au Nunavut à raison de 119,2 cas pour 100000 habitants en 2015, il était près de la moitié de ce qu'il était en 2014. Une éclosion à Terre-Neuve-et-Labrador a donné lieu à une augmentation notable du nombre de cas déclarés et du taux d'incidence dans cette province. En 2015, les hommes représentaient un peu plus de la moitié des cas déclarés, soit $53 \%$, et les Canadiens plus âgés représentaient le plus lourd fardeau de la tuberculose, avec un taux d'incidence de 10,3 cas pour 100000 habitants. Les personnes nées à l'étranger ont continué de représenter la majorité des cas déclarés à raison de $71 \%$, mais le taux d'incidence chez les Autochtones nés au Canada est resté le plus élevé à raison 17,1 cas pour 100000 habitants, particulièrement au sein de la population inuite à raison de 166,2 cas pour 100000 habitants. La tuberculose pulmonaire est demeurée la maladie déclarée le plus souvent. Les données sur les résultats des traitements des cas déclarés en 2014 ont indiqué que $85 \%$ des cas avaient été guéris ou avaient terminé leur traitement.

Conclusion : Les taux de tuberculose au Canada n'ont que très peu changé au cours de la dernière décennie et demeurent faibles et stables dans l'ensemble. Cependant, les personnes nées à l'étranger et les Autochtones canadiens étaient toujours représentés de façon disproportionnée parmi les cas déclarés de tuberculose en 2015. En tant que principale source de données nationales sur les cas de tuberculose au Canada, les données contenues dans le présent rapport fournissent des renseignements opportuns pour les mesures de santé publique, ainsi que pour l'élaboration et l'évaluation des politiques et des programmes.

\begin{abstract}
Affiliation
${ }^{1}$ Centre de la lutte contre les maladies transmissibles et les infections, Agence de la santé publique du Canada, Ottawa (Ontario)
\end{abstract}

*Correspondance : $\mathrm{tb}$ surveillance@phac-aspc.gc.ca

Citation proposée : Gallant V, Duvvuri V, McGuire M. La tuberculose au Canada - Résumé 2015. Relevé des maladies transmissibles au Canada. 2017;43(3):85-91. https://doi.org/10.14745/ccdr.v43i34a04f

\section{Introduction}

La tuberculose est un important problème de santé mondial. En 2015, on estimait à 10,4 millions le nombre de nouveaux cas (incidents), ce qui correspond à un taux d'incidence global de 142 cas pour 100000 habitants (1). Même si le taux d'incidence de la tuberculose progressive dans la population canadienne diminue d'une année à l'autre et est l'un des plus faibles au monde, des taux élevés persistent chez les Autochtones et les personnes nées à l'étranger $(2,3)$.

Le portefeuille fédéral de la Santé, en partenariat avec les gouvernements provinciaux et territoriaux et d'autres ministères et organismes fédéraux, s'emploie à lutter contre la tuberculose au Canada. L'Agence de la santé publique du Canada (ASPC) assure la surveillance de la tuberculose progressive au moyen du Système canadien de déclaration des cas de tuberculose (SCDCT), en collaboration avec les ministères de la Santé des provinces et des territoires. L'ASPC utilise les données de surveillance de la tuberculose pour observer les progrès réalisés vers l'atteinte de l'objectif que s'est fixé le Canada, soit de prévenir et de contrôler la transmission de la tuberculose, tel qu'il est décrit dans le document intitulé Prévention et contrôle de la tuberculose au Canada - Un cadre d'action fédéral (4). 
Ce résumé présente un aperçu descriptif de tous les cas de tuberculose progressive (nouveaux cas et cas de retraitement) au Canada en 2015, selon la répartition géographique, l'âge, le sexe, l'origine et la classification des diagnostics. Les résultats sont discutés en fonction du contexte des données des années précédentes. Les résultats du traitement des cas diagnostiqués en 2014 sont également présentés.

Auparavant, ces données étaient publiées chaque année dans un document distinct intitulé La tuberculose au Canada: Prédiffusion. II s'agit de la première version de ce rapport à être publiée sous un nouveau titre : La tuberculose au Canada : Résumé 2015, contenue dans le Relevé des maladies transmissibles au Canada (RMTC). Des données supplémentaires sont disponibles en ligne (5).

\section{Méthodologie}

Le SCDCT est un système de surveillance fondé sur les cas qui conserve certaines données non nominatives sur les personnes diagnostiquées d'une tuberculose progressive. Les détails relatifs aux méthodes du système, y compris les processus de collecte des données, la gestion des données, le contrôle de la qualité des données, l'analyse ainsi que la classification et la catégorisation des sous-groupes de population ont déjà été décrits (2). En bref, les autorités de santé publique provinciales et territoriales soumettent volontairement leurs données sur les nouveaux cas et les cas de retraitement qui répondent à la définition canadienne des cas faisant l'objet d'une surveillance nationale (5). Les données sur les résultats des traitements sont soumises entre 12 et 18 mois suivant la soumission du rapport de cas initial. Si le traitement est en cours au moment de la soumission des données à l'ASPC, le territoire de compétence qui effectue la déclaration soumet un rapport intérimaire, suivi de mises à jour annuelles, jusqu'à ce que le dossier soit fermé.

Les données sont soumises à l'ASPC au moyen d'un formulaire de déclaration standard rempli manuellement ou transmis électroniquement. Toutes les données brutes (formulaires papier et électroniques) sont conservées conformément à la directive de l'Agence sur la collecte, l'utilisation et la diffusion de l'information sur la santé publique (Agence de la santé publique du Canada. 2013 [document non publié]).

Le «taux d'incidence » fait référence au nombre de personnes ayant reçu un diagnostic de tuberculose progressive (nouveaux cas et cas de retraitement) pour 100000 habitants pour chaque année de déclaration. Les dénominateurs des populations utilisés pour calculer les taux proviennent de plusieurs différentes sources. Les taux relatifs aux dénombrements des populations provinciales, territoriales ou de l'ensemble du pays par âge et par sexe sont fondés sur les estimations de mi-année tirées des données du recensement de 2011 et produites par la Division de la démographie de Statistique Canada (données non publiées). Le dénombrement des personnes nées à l'étranger est évalué à partir de l'enquête par sondage auprès des ménages de 2011 (6). Pour les groupes autochtones, les Premières nations, les Inuits et les Métis, les taux sont également fondés sur les données de l'Enquête auprès des ménages de 2011 (7). Enfin, les taux relatifs aux membres des Premières nations inscrits, vivant dans les réserves et hors réserve, ont été calculés en utilisant les projections de population produites par Affaires autochtones et du Nord Canada (données non publiées).

Les logiciels Microsoft Excel 2010 et SAS Enterprise Guide (SAS EG) v5.1 ont été utilisés pour le nettoyage et l'analyse des données. Aucune procédure statistique n'a été utilisée pour les analyses comparatives, et aucune technique statistique n'a été appliquée pour tenir compte des données manquantes. À l'exception des données sur les facteurs de risque, les données recueillies à l'aide de ce système sont très complètes. Les données des tableaux contenant de petites cellules $(n=<5)$ $n$ 'ont pas été supprimées puisqu'elles ne semblaient pas présenter de risque d'identification de cas individuels. Ces procédures sont conformes à la directive de l'Agence sur la collecte, I'utilisation et la diffusion de l'information sur la santé publique (document non publié). Les données ont été approuvées par les provinces et les territoires afin d'en assurer l'exactitude.

Les données du présent rapport ont été extraites du SCDCT en août 2016. Les cas de tuberculose sont pris en compte à partir de la date à laquelle le territoire de compétence qui effectue la déclaration confirme le diagnostic de tuberculose. Étant donné que les données à l'échelle nationale sont soumises annuellement, toute mise à jour est généralement soumise 12 mois après la soumission annuelle initiale.

\section{Résultats}

Au Canada, 1639 cas de tuberculose progressive ont été déclarés en 2015, ce qui représente une légère augmentation du nombre de cas déclarés en 2014 (1 614) et une augmentation correspondante du taux d'incidence de 4,5 cas pour 100000 habitants à 4,6 cas pour 100000 habitants (figure 1, tableau complémentaire 1A [5]). Des cas déclarés en 2015, $92 \%$ (1509) ont été désignés comme des nouveaux cas. Six pour cent (101) des cas ont été déclarés comme des cas de retraitement, et aucun antécédent de tuberculose n'était connu pour 2 \% (29) des cas déclarés (données non présentées).

Figure $1:$ Nombre de cas de tuberculose progressive déclarés (nouveaux cas et cas de retraitement) et taux d'incidence au Canada pour 100000 habitants, de 2005 à 2015

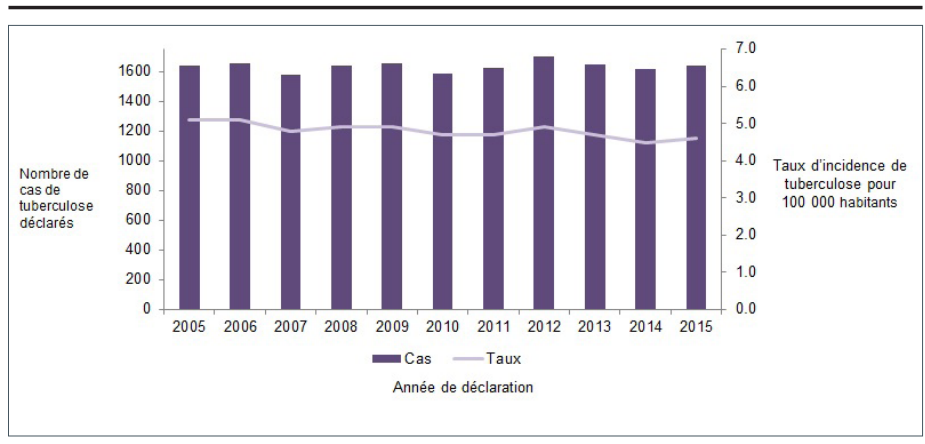

\section{Répartition géographique}

En 2015, les taux d'incidence de la tuberculose dans les provinces de l'Atlantique (Nouveau-Brunswick, Nouvelle-Écosse et Île-du-Prince-Édouard), en Ontario et au Québec étaient inférieurs au taux d'incidence national, qui était de 4,6 cas pour 100000 habitants (figure 2). Les taux d'incidence dans toutes les autres provinces et tous les autres territoires étaient plus élevés que le taux canadien.

Le Nunavut a continué d'avoir le plus haut taux d'incidence de la tuberculose à raison de 119,2 cas pour 100000 habitants, soit 
un taux qui était 26 fois plus élevé que le taux global canadien. Toutefois, ce taux d'incidence était de près de la moitié de ce qu'il était en 2014, c'est-à-dire 232,8 cas pour 100000 habitants. En raison d'une éclosion de tuberculose, le nombre de cas déclarés dans la province de Terre-Neuve-et-Labrador a augmenté de sept cas en 2014 à 33 cas en 2015, ce qui quintuple le taux d'incidence de la tuberculose dans cette province (6,3 cas par rapport à 1,3 cas pour 100000 habitants). Des données supplémentaires sont fournies dans le tableau complémentaire 1A (5).

Figure 2 : Taux d'incidence de la tuberculose au Canada en 2015 pour 100000 habitants, par province ou territoire

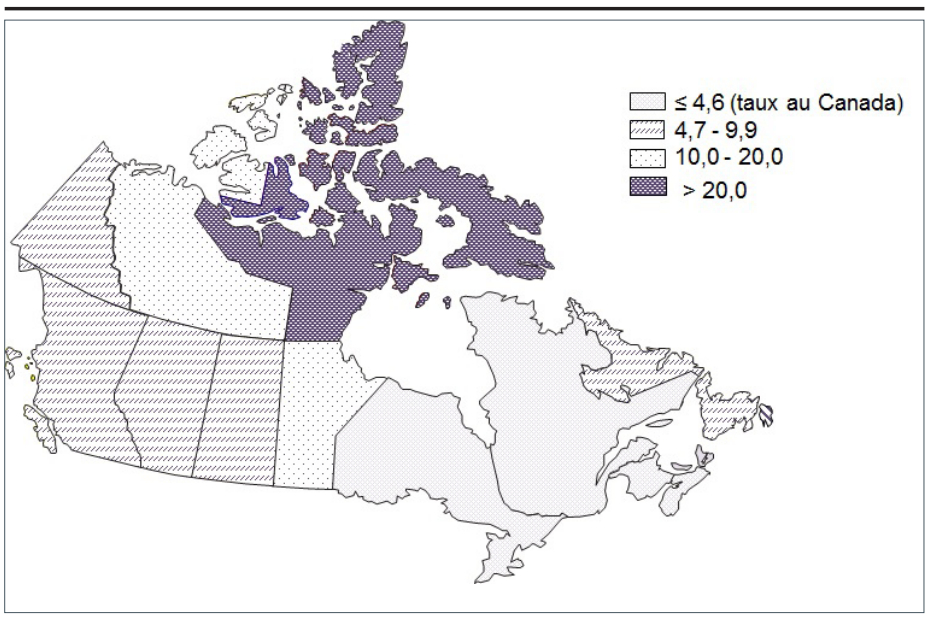

\section{Répartition par âge et par sexe}

Au Canada, entre 2005 et 2015, les hommes comptaient pour un pourcentage sensiblement plus élevé de cas déclarés que les femmes avec un taux d'incidence proportionnellement plus élevé (figure 3). En 2015, les hommes représentaient $53 \%$ (871 cas) des cas déclarés, ce qui correspond à un taux d'incidence de 4,9 cas pour 100000 habitants. À titre de comparaison, les femmes représentaient $47 \%$ (768 cas) de tous les cas déclarés, ce qui correspond à un taux d'incidence de 4,2 cas pour 100000 habitants (tableaux complémentaires 1B et 1C [5]).

Figure 3 : Nombre de cas de tuberculose progressive déclarés (nouveaux cas et cas de retraitement) et taux d'incidence au Canada pour 100000 habitants, selon le sexe, de 2005 à 2015

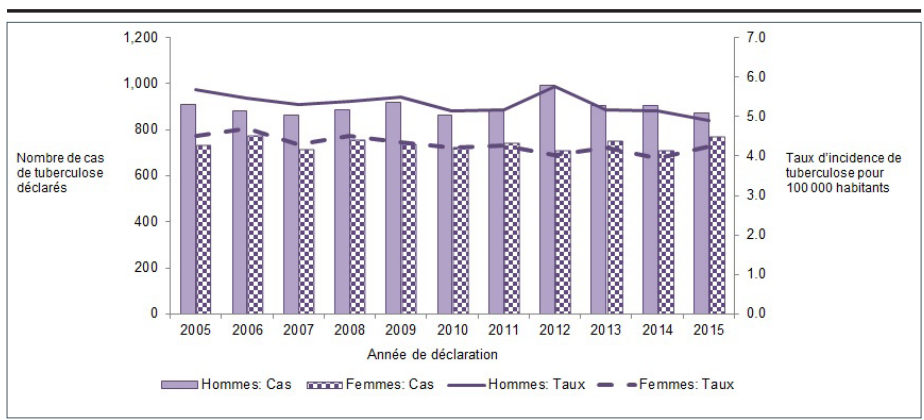

De 2005 à 2015, les taux d'incidence de la tuberculose sont demeurés stables ou ont diminué légèrement pour tous les groupes d'âge (tableau complémentaire 2 [5]; figure 4). Les taux d'incidence ont diminué de façon la plus appréciable dans le groupe d'âge de 65 à 74 ans; ils sont passés de 7,5 cas pour
100000 habitants en 2005 à 5,2 cas pour 100000 habitants en 2014. Comme au cours des années précédentes, en 2015, les personnes âgées (75 ans et plus) représentaient le plus important fardeau de la maladie, avec un taux d'incidence de 10,3 cas pour 100000 habitants. Se reporter aux données du tableau complémentaire 2 (5).

Figure 4 : Taux d'incidence de la tuberculose au Canada pour 100000 habitants, par groupe d'âge, de 2005 à 2015

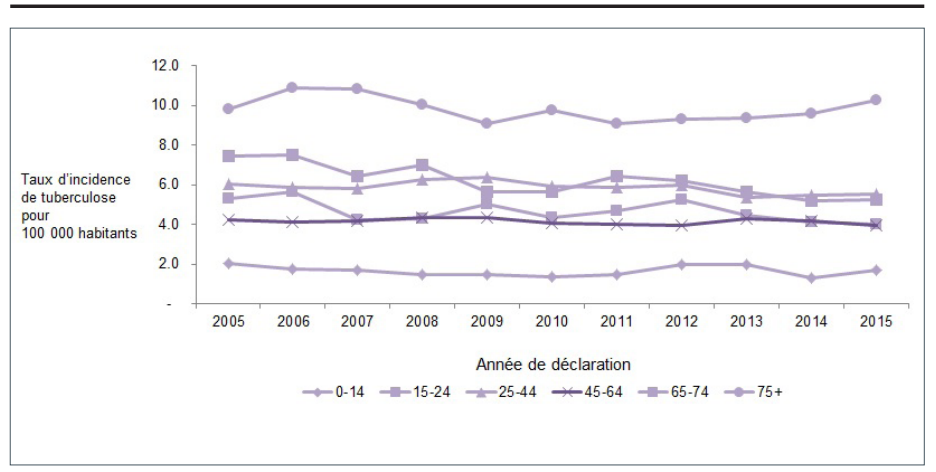

En 2015, les personnes âgées de 25 à 44 ans ont continué de représenter le plus fort pourcentage de cas déclarés, soit $33 \%$ (535) (figure 5), et six pour cent de tous les cas déclarés (97) ont été observés chez des enfants de moins de 15 ans. La tuberculose chez les très jeunes enfants indique souvent une transmission récente de la maladie (8) (figure 5). Au Manitoba, au Nunavut, à Terre-Neuve-et-Labrador et au Québec, plus de $10 \%$ des cas déclarés étaient des enfants de moins de 15 ans (figure 5, tableau complémentaire 3 [5]).

Figure 5 : Répartition des cas de tuberculose progressive (nouveaux cas et cas de retraitement) par groupe d'âge et par province ou territoire et dans l'ensemble du Canada, en 2015

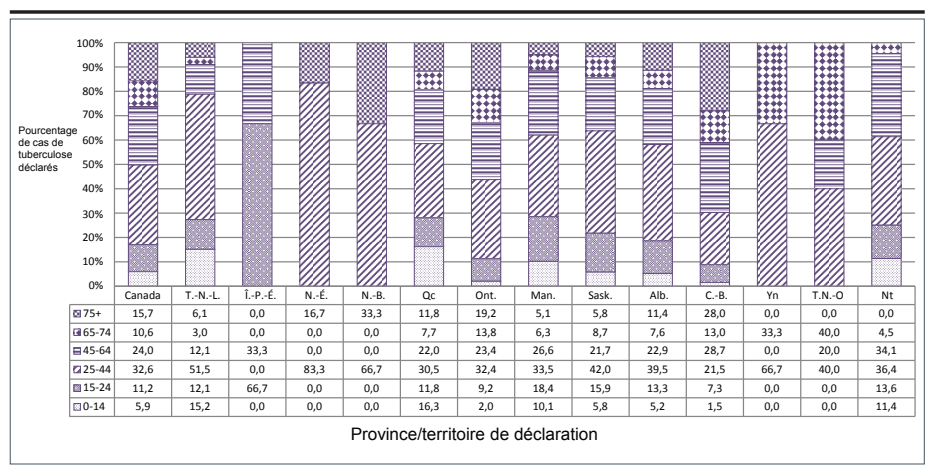

Abréviations : Alb., Alberta; C.-B., Colombie-Britannique; Man., Manitoba; N.-B.,

Nouveau-Brunswick; T.-N.-L., Terre-Neuve-et-Labrador; N.-É., Nouvelle-Écosse; Nt, Nunavut; T.N.-O., Territoires du Nord-Ouest; Ont., Ontario; Î.-P.-É., île-du-Prince-Édouard; Qc, Québec Sask., Saskatchewan; Yn, Yukon

\section{Répartition selon l'origine}

Les personnes nées à l'étranger et les Autochtones nés au Canada étaient toujours représentés de façon disproportionnée parmi les cas déclarés de tuberculose en 2015 (figure 6). Les personnes nées à l'étranger, qui composaient environ $22 \%$ de la population canadienne totale en 2015, représentaient $71 \%$ (1 169 sur 1639 ) de tous les cas déclarés avec un taux d'incidence de 14,8 cas pour 100000 habitants. Les Autochtones 
nés au Canada composaient $5 \%$ de la population canadienne totale en 2015 , mais représentaient $17 \%$ (281 sur 1639$)$ de tous les cas déclarés, avec un taux d'incidence de 17,1 cas pour 100000 habitants. Les non-Autochtones nés au Canada représentaient le plus faible pourcentage de cas déclarés, soit $11 \%$ (170 sur 1639 ), avec un taux d'incidence de 0,6 cas pour 100000 habitants. L'origine était inconnue dans un pourcent (19 sur 1639 ) des cas signalés (figure 6, tableau complémentaire 4 [5]).

Figure 6 : Répartition des cas de tuberculose progressive (nouveaux cas et cas de retraitement) au Canada par origine en 2015

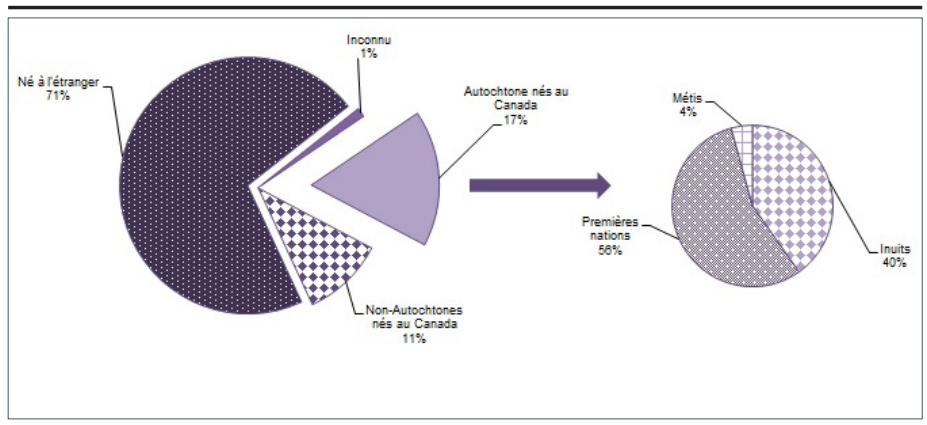

La répartition des cas de tuberculose entre les groupes d'origine variait selon la province et le territoire. Au Manitoba, à Terre-Neuve-et-Labrador et dans le Nord, au nord (Territoires du Nord-Ouest, Nunavut et Yukon) et en Saskatchewan, la plupart des cas étaient des Autochtones nés au Canada. En Alberta, en Colombie-Britannique, dans les provinces de l'Atlantique (Nouveau-Brunswick, Nouvelle-Écosse et Île-du-Prince-Édouard), en Ontario et au Québec, la plupart des cas déclarés étaient des personnes nées à l'étranger. Au Nouveau-Brunswick et au Québec, environ $30 \%$ des cas déclarés étaient des non-Autochtones nés au Canada. Pour le reste des provinces et des territoires, les cas non-autochtones nés au Canada représentaient moins de $10 \%$ des cas signalés (tableau complémentaire 4 [5]).

\section{Autochtones}

Les Autochtones nés au Canada sont répartis dans trois populations distinctes : les Premières nations, les Inuits et les Métis. En 2015, des 281 cas déclarés parmi les Autochtones nés au Canada, $56 \%$ (156) étaient des membres des Premières nations, $40 \%$ (113) étaient Inuits et $4 \%$ (12) étaient Métis (figure 6).

En 2015, comparativement au taux d'incidence de 0,6 cas pour 100000 habitants dans la population non-autochtone née au Canada, le taux d'incidence parmi les Métis (2,2 cas pour 100000 habitants) était près de quatre fois plus élevé, et le taux d'incidence chez les membres des Premières nations (15,1 cas pour 100000 habitants) était 25 fois plus élevé. Le taux d'incidence le plus élevé de tous les groupes d'origine a été observé chez les Inuits, à raison de 166,2 cas pour 100000 habitants, soit un taux qui était plus de 270 fois plus élevé que le taux pour l'ensemble des non-Autochtones nés au Canada (figure 7).
Figure 7 : Taux d'incidence de la tuberculose pour 100000 habitants par origine au Canada en 2015

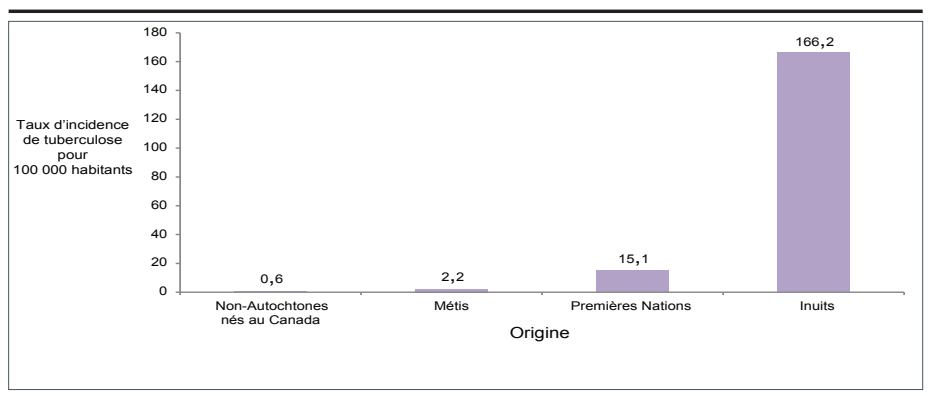

\section{Cas des personnes nées à l'étranger par région et par pays de naissance}

En 2015, parmi les personnes nées à l'étranger, 1169 cas de tuberculose ont été déclarés au Canada, ce qui représente un taux d'incidence de 14,8 cas pour 100000 habitants, soit un taux neuf fois plus élevé que le taux de la population née au Canada (1,6 cas pour 100000 habitants).

En fonction de leur pays de naissance, les cas d'origine étrangère étaient regroupés dans l'une des neuf régions épidémiologiques définies par le Partenariat Halte à la tuberculose et l'Organisation mondiale de la Santé (9). Des 1169 cas de tuberculose chez les personnes nées à l'étranger en 2015, 42 \% (495) sont nées dans la région du Pacifique occidental et $26 \%$ (303) sont nées dans des régions du sud-est de l'Asie. La plupart des cas nés à l'étranger diagnostiqués au Canada avaient pour pays de naissance un pays considéré par l'Organisation mondiale de la Santé comme fortement atteint par la tuberculose. Environ $60 \%$ de tous les cas nés à l'étranger étaient nés aux Philippines, en Inde, en Chine, au Vietnam et au Pakistan (données non présentées). En 2015, le taux d'incidence le plus élevé, soit 41,5 cas pour 100000 habitants, a été observé chez les personnes nées en Afrique où la prévalence du VIH est élevée (tableau complémentaire 4 [5]).

\section{Classification des diagnostics}

La tuberculose active peut être classée comme maladie respiratoire ou maladie non respiratoire. La tuberculose respiratoire comprend la tuberculose pulmonaire, la tuberculose pleurale, la tuberculose des ganglions lymphatiques endothoraciques ou des ganglions lymphatiques du médiastin, ainsi que la tuberculose du larynx, du nasopharynx, du nez et des sinus. À la forme primaire de la maladie, l'état morbide caractérisé par une pleurite et un épanchement pleural (habituellement chez un adolescent ou un jeune adulte, mais possiblement dans n'importe quel groupe d'âge, découlant d'une infection récente [dans les 24 mois précédents] par le complexe Mycobacterium tuberculosis) est également classé dans la catégorie des maladies respiratoires. La tuberculose non respiratoire désigne tous les autres sièges de la maladie.

Entre 2005 et 2015, la tuberculose respiratoire représentait $76 \%$ (13 745 sur 17 975) de tous les cas diagnostiqués de tuberculose au Canada (tableau complémentaire 5 [5]). En 2015, 66 \% des cas déclarés ont reçu un diagnostic de tuberculose pulmonaire. La tuberculose pulmonaire figurait parmi la classification de 
Figure 8 : Répartition des cas de tuberculose déclarés par classification du diagnostic principal au Canada, dans les provinces et dans les territoires, en 2015

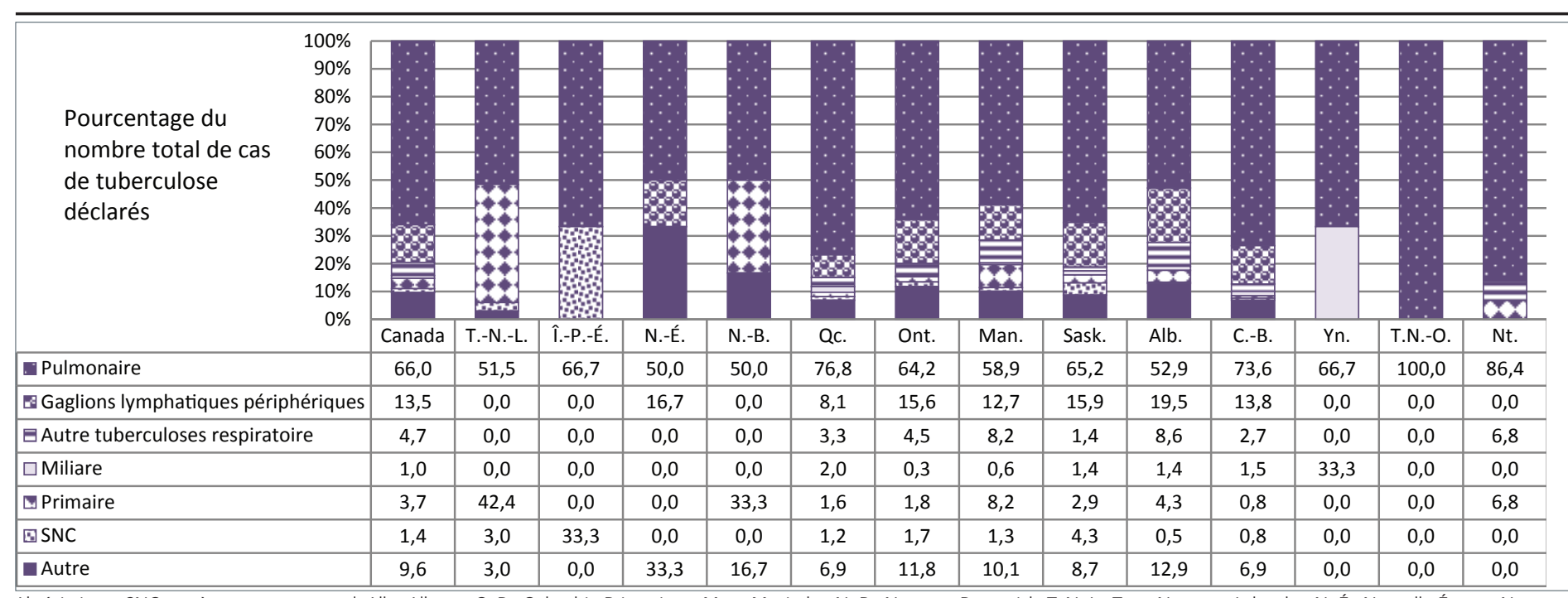

Abréviations : SNC, système nerveux central; Alb., Alberta; C.-B., Colombie-Britannique; Man., Manitoba; N.-B., Nouveau-Brunswick; T.-N.-L., Terre-Neuve-et-Labrador; N.-É., Nouvelle-Écosse; Nt,

Nunavut; T.N.-O., Territoires du Nord-Ouest; Ont., Ontario; Î.-P.-É., Île-du-Prince-Édouard; Qc, Québec; Sask., Saskatchewan; Yn, Yukon

diagnostic la plus souvent déclarée dans toutes les provinces et tous les territoires. La tuberculose des ganglions lymphatiques périphériques est demeurée la deuxième localisation la plus souvent déclarée. Dans l'ensemble, $4 \%$ (60) des cas déclarés ont reçu un diagnostic de primo-infection tuberculeuse. Toutefois, $42 \%$ (1 433) des cas déclarés par Terre-Neuve-et-Labrador ont reçu un diagnostic de primo-infection tuberculeuse (figure 8 ). Se reporter aux données du tableau complémentaire 6 (5).

\section{Résultats des traitements pour 2014}

Les résultats des traitements étaient disponibles dans $97 \%$ (1 562 sur 1614 ) de tous les cas de tuberculose progressive en 2014 (tableau complémentaire 7 [5]). Sur les cas signalés pour lesquels des données relatives aux résultats des traitements étaient disponibles :

- 85 \% (1 328) ont été guéris ou avaient terminé leur traitement;

- huit pourcent (119) sont décédés avant ou pendant le traitement;

- deux pourcent (28) avaient quitté le territoire de compétence avant d'avoir terminé leur traitement;

- moins de un pourcent (11) ont été classés dans une autre catégorie de résultats (quatre cas de non-observance, trois cas de refus de traitement, un cas d'abandon du traitement en raison d'une grossesse, et les résultats des trois cas restants n'ont pas été précisés);

- moins de un pourcent (13) ont été perdus de vue;

- moins de un pourcent (9) ont arrêté le traitement en raison d'effets indésirables; et

- trois pourcent (54) ont été déclarés comme en cours de traitement.

\section{Discussion}

Le nombre de cas de tuberculose déclarés chaque année au Canada est demeuré relativement stable depuis 2005, s'élevant à environ 1630 cas par année. En 2015, 1639 cas de tuberculose progressive ont été déclarés, ce qui représente une légère augmentation du nombre de cas déclarés en 2014 (1 614) et une augmentation correspondante du taux d'incidence de 4,5 cas pour 100000 habitants à 4,6 cas pour 100000 habitants. Bien que le taux d'incidence de la tuberculose demeure le plus élevé au Nunavut, le nombre de cas déclarés et le taux d'incidence global ont nettement diminué en 2015. Par contre, Terre-Neuve-et-Labrador a connu une éclosion importante de tuberculose en 2015, ce qui a donné lieu à une augmentation du nombre de cas de tuberculose déclarés et une augmentation correspondante du taux d'incidence global. Une surveillance continue est nécessaire afin de déterminer si ces changements indiquent une tendance qui se maintiendra.

Dans l'ensemble, aucun changement n'a été relevé quant à la répartition des cas par groupe d'âge, par sexe ou par siège de maladie diagnostiqué. En 2015, les personnes nées à l'étranger ont continué de représenter la majorité des cas de tuberculose déclarés, mais le taux d'incidence est resté le plus élevé chez les Autochtones nés au Canada et, en particulier, au sein de la population inuite. La tuberculose pulmonaire est restée la maladie la plus souvent signalée en 2015 , et les données sur les résultats de traitement disponibles pour les cas déclarés en 2014 ont indiqué que $82,3 \%$ des cas avaient été guéris ou avaient terminé leur traitement.

Les données contenues dans le présent rapport sont considérées comme provisoires et sont susceptibles de changer dans les publications à venir de la série de rapports de surveillance La tuberculose au Canada. Les différences entre les données publiées dans le présent rapport et les données publiées dans 
les rapports de surveillance nationaux, provinciaux et territoriaux précédents peuvent s'expliquer par des retards de déclaration ou des différences relatives au moment où les données ont été extraites des diverses bases de données de surveillance. La province ou le territoire peut mettre à jour ses données publiées plus régulièrement. En cas de différences entre les données du présent rapport et les données des rapports provinciaux ou territoriaux, les lecteurs sont invités à communiquer avec les gouvernements provinciaux et territoriaux pour obtenir des clarifications.

Les divers rapports ont des limites dont il faut tenir compte. Étant donné que le SCDCT est un système de surveillance passif, il repose sur les données recueillies de façon rétrospective à partir des dossiers médicaux et des résultats de laboratoire et non pas sur les données de cas actifs sollicités. Par conséquent, il est difficile de déterminer si toutes les personnes atteintes de tuberculose progressive ont été identifiées et déclarées. Toutefois, l'Organisation mondiale de la Santé estime que le système de surveillance du Canada a un taux de détection des cas de $90 \%$ et que l'étendue de l'incertitude est de $78 \%$ à $100 \%$ (1). L'exactitude des données dépend, en partie, du moment où les provinces et territoires communiquent leurs déclarations et mises à jour à l'ASPC. II existe un certain décalage (i.e. créant un retard de déclaration). Comme pour toutes les données de surveillance, les données du présent rapport peuvent présenter des erreurs occasionnelles de codage, de déclaration ou de traitement. Plus de $95 \%$ des éléments de données sont complets pour la plupart des données démographiques et cliniques.

En comparaison avec les autres pays du G7 (France, Allemagne, Grande-Bretagne, Italie, Japon et États-Unis), le Canada a le deuxième plus faible taux de tuberculose après les États-Unis d'Amérique (1). Les mises à jour annuelles sur le nombre de cas de tuberculose progressive au Canada et les taux d'incidence correspondants sont importantes dans le suivi des progrès réalisés en ce qui concerne l'objectif de réduction du fardeau de la tuberculose au Canada. En tant que principale source de données nationales sur les cas de tuberculose au Canada, les données contenues dans le présent rapport fournissent des renseignements opportuns pour les mesures de santé publique, ainsi que pour l'élaboration et l'évaluation des politiques et des programmes.

\section{Remerciements}

L'Agence de la santé publique du Canada tient à remercier les personnes des programmes provinciaux et territoriaux de lutte antituberculeuse suivantes de leur contribution et de leur participation au SCDCT.

Brenda P. Earles, Health and Community Services, Population Health Branch, Terre-Neuve-et-Labrador

Jennifer Phillips, Health and Community Services, Population Health Branch, Terre-Neuve-et-Labrador

Carolyn Sandford, ministère de la Santé et du Mieux-être de I'Île-du-Prince-Édouard

Stacey Burns, ministère de la Santé et du Mieux-être de I'île-du-Prince-Édouard

Beverly A. Billard, ministère de la Santé et du Mieux-être de la Nouvelle-Écosse.
Suzanne Savoie, ministère de la Santé du Nouveau-Brunswick Hanan Smadi, ministère de la Santé du Nouveau-Brunswick Paul Rivest, Direction régionale de santé publique de Montréal, Québec

Maria-Constanza Street, Direction régionale de santé publique de Montréal, Québec

Michael Whelan, Santé publique Ontario

Cecilia Fung, Santé publique Ontario

Robert Wang, Santé Manitoba

Sharon Fletcher, Santé Manitoba

Helen Bangura, ministère de la Santé de la Saskatchewan

Valerie Mann, Ministère de la Santé de la Saskatchewan

Assaad Al-Azem, TB Prevention and Control Saskatchewan

Rosa Maheden, ministère de la Santé de l'Alberta

Celine O'Brien, ministère de la Santé de l'Alberta

Myrna Fleischauer, Alberta Health Services

Faye Hutton, Centre de contrôle des maladies de la

Colombie-Britannique

Gloria Mui, Centre de contrôle des maladies de la

Colombie-Britannique

David Roth, Centre de contrôle des maladies de la

Colombie-Britannique

Beth Roberts, Yukon Communicable Disease Control

Beth Roberts, Centre de lutte contre les maladies transmissibles du Yukon

Caroline Newberry, ministère de la Santé et des Services sociaux des Territoires du Nord-Ouest

Karen Hollett, ministère de la Santé et des Services sociaux des Territoires du Nord-Ouest

Elaine Randell, ministère de la Santé du Nunavut

\section{Conflit d'intérêts}

Aucun.

\section{Financement}

Ce travail a été appuyé par l'Agence de la santé publique du Canada dans le cadre de son mandat de base.

\section{Références}

1. World Health Organization. Global tuberculosis report 2016. WHO/HTM/TB/2016.13.22. Geneva: WHO; 2016.

2. Agence de la santé publique du Canada. La tuberculose au Canada 2012 [Internet]. Ottawa: ASPC; 2015 [mise à jour le 29 mai 2015; consulté le 5 fév 2017]. Disponible à l'adresse : http://www.phac-aspc.gc.ca/tbpc-latb/pubs/tbcan12/indexfra.php.

3. Gouvernement du Canada. La tuberculose au Canada 2014 : Prédiffusion [Internet]. Ottawa: ASPC; 2016 [mise à jour le 15 mars 2016; consulté le 5 fév 2017]. Disponible à l'adresse : https://www.canada.ca/fr/sante-publique/services/ publications/maladies-et-affections/tuberculose-canada2014-prediffusion.html.

4. Agence de la santé publique du Canada. Prévention et contrôle de la tuberculose au Canada. Un cadre d'action fédéral [Internet]. Ottawa: ASPC; 2014 [mise à jour le 22 mars 2014; consulté le 5 fév 2017]. Disponible à l'adresse : http://www.phac-aspc.gc.ca/tbpc-latb/pubs/tpc-pct/indexfra.php. 
5. Gallant V, Duvvuri V, McGuire M. Tuberculose au Canada : Données supplémentaire de 2015 [Internet]. Relevé des maladies transmissibles au Canada. 2017;43(3) Disponible à l'adresse : http://www.phac-aspc.gc.ca/publicat/ccdrrmtc/17vol43/dr-rm43-3-4/ar-05-fra.php.

6. Statistique Canada. 2011 enquêtes auprès des ménages. Catalogue numéro. 99-010-x20111026 [Internet]. Ottawa: Statistique Canada; 2012 [Mise à jour le 5 jan 2017; consulté le 5 fév 2017]. Disponible à l'adresse : http://www12.statcan. gc.ca/census-recensement/index-fra.cfm.

7. Statistique Canada. Projections de la population et des ménages autochtones au Canada, 2011 à 2036. Catalogue no. 91-552 [Internet]. Ottawa: Statistique Canada; 2015.
Disponible à l'adresse : http://www.statcan.gc.ca/pub/91552-x/91-552-x2015001-fra.pdf.

8. Agence de la santé publique du Canada. Normes canadiennes pour la lutte antituberculeuse 7e édition [Internet]. Ottawa : Société canadienne de thoracologie et l'Agence de la santé publique du Canada; 2013 [mise à jour le 17 fév 2014; consulté le 5 fév 2017]. Disponible à l'adresse : http://www.phac-aspc.gc.ca/tbpc-latb/pubs/tbcanada-7/index-fra.php.

9. Stop TB Partnership and World Health Organization. The global plan to stop TB 2006-2015 [Internet]. Geneva: WHO; 2006. Disponible à l'adresse : http://www.stoptb.org/assets/ documents/global/plan/GlobalPlanFinal.pdf.
RESPIREZ

l'association pulmonaire

\section{La ressource ultime pour le diagnostic et le traitement :}

\section{Normes canadiennes pour la lutte antituberculeuse $7^{e}$ édition}

PDF gratuit et disponible pour le téléchargement

Recherche Web : Normes canadiennes antituberculeuses 